\title{
A VARIATIONAL METHOD FOR THE CONSTRUCTION OF CONVERGENT ITERATIVE SEQUENCES
}

\author{
ZALMAN RUBINSTEIN
}

(Received 20 June 1984; revised 3 September 1984)

Communicated by J. H. Chabrowski

\begin{abstract}
Convergent iterative sequences are constructed for the polynomials $f_{m}=z+z^{m}, m \geqslant 2$, with initial point the lemniscate $\left\{z:\left|f_{m}^{\prime}(z)\right| \leqslant 1\right\}$. In the particular case $m=2$ convergent iterative sequences are constructed also for $f_{m}^{-1}(z)$ with an arbitrary initial point. The method is based on a certain variational principle which allows reducing the problem to the well known situation of an analytic function mapping a simply connected domain into a proper subset of itself and possessing a fixed point in the domain.
\end{abstract}

1980 Mathematics subject classification (Amer. Math. Soc.): 30 C 10; secondary 39 B 10.

\section{Introduction}

The following easy consequence of Schwarz's lemma and the Riemann mapping theorem was applied in [3] for the construction of convergent iterative radicals.

LEMMA 1. Let $f$ be an analytic mapping of a simply connected region $G$ of the complex plane into one of its proper subsets. If $f$ has a fixed point $p \in G$, then for every $z_{0} \in G$ the sequence $z_{n+1}=f\left(z_{n}\right), n=0,1, \ldots$, converges to $p$ as $n \rightarrow \infty$.

The restriction of $p \in G$ is essential for the proof of Lemma 1. However, in many applications it appears that $p$ is on the boundary of $G$. We then apply Lemma 1 to a perturbed function $f_{\varepsilon}$ which depends on a positive parameter $\varepsilon$ and

(ㄷ) 1986 Australian Mathematical Society $0263-6115 / 86 \$ A 2.00+0.00$ 
show that the perturbed sequences $\left\{w_{n}(\varepsilon)\right\}_{n=0}^{\infty}, w_{n}(\varepsilon)=f_{\varepsilon}\left(w_{n-1}(\varepsilon)\right)$, converge to $\left\{z_{n}\right\}_{n=0}^{\infty}$ as $\varepsilon \rightarrow 0$ eventually uniformly in $n$ (see Lemma 3 ). We apply this procedure to the construction of a convergent iterative polynomial sequence of arbitrary degree $m(m \geqslant 2)$, where $f=z+z^{m}$, and where $G$ is a component of the lemniscate $\left\{z:\left|f^{\prime}(z)\right|<1\right\}$. In the particular case $m=2$ the variational method is also applied to an analytic branch of $f^{-1}$ in a suitable $G$ to construct convergent sequences with an arbitrary $z_{0}$. Certain open problems are mentioned. For a general existence theorem of convergent cyclic sequences formed by $f$ and $f^{-1}$, see [1].

\section{Several lemmas}

Throughout this note $f(z)=z+z^{m}, m \geqslant 2$, and $R=\left\{z:\left|f^{\prime}(z)\right|<1\right\} ; R$ consists of $m-1$ simply connected components having a joint boundary point at the origin. Each component has two tangents at the origin which make an angle of $\alpha=\pi /(m-1)$. Two adjacent components are separated by a sector of aperture $\alpha$. Let $R_{m}$ be the component of $R$ which is symmetric with respect to the ray $\arg z=\alpha$.

LeMma 2. $f(z): R_{m} \rightarrow R_{m}$.

Proof. (a) We show first $f(z): R \rightarrow R$. We have to show that for $z \in R$

$$
\left|\left(z+z^{m}\right)^{m-1}+\frac{1}{m}\right|<\frac{1}{m},
$$

or, setting $w=1 / m+z^{m-1}$, that

$$
h(w)=\left(w-\frac{1}{m}\right)\left(w+1-\frac{1}{m}\right)^{m-1}+\frac{1}{m}
$$

has modulus less than $1 / m$ for $|w|<1 / m$. Now

$$
\begin{aligned}
h(w)= & \sum_{k=2}^{m} \frac{(m-1)^{m-k-1}}{m^{m-k}}\left[(m-1)\left(\begin{array}{c}
m-1 \\
k-1
\end{array}\right)-\left(\begin{array}{c}
m-1 \\
k
\end{array}\right)\right] w^{k} \\
& +\frac{1}{m}\left[1-\left(\frac{m-1}{m}\right)^{m-1}\right] .
\end{aligned}
$$

Denote the first sum by $h_{1}(w) . h_{1}(w)$ has positive coefficients, so that $\left|h_{1}(w)\right|<$ $h_{1}(1 / m)$ for $|w|<1 / m$. Now a direct calculation shows that

$$
h_{1}\left(\frac{1}{m}\right)=\frac{(m-1)^{m-1}}{m^{m}} \text {. }
$$


Therefore,

$$
|h(w)|<h_{1}\left(\frac{1}{m}\right)+\frac{1}{m}-\frac{(m-1)^{m-1}}{m^{m}}=\frac{1}{m} .
$$

(b) Now let $z \in R_{m}$. Then

$$
\arg f(z)=\arg z+\arg \left(1+z^{m-1}\right) .
$$

Also,

$$
1+z^{m-1}=\frac{m-1}{m}+\rho e^{i \phi}, \quad 0 \leqslant \rho \leqslant \frac{1}{m},
$$

so that $\theta=\arg \left(1+z^{m-1}\right)$ satisfies $|\operatorname{tg} \theta| \leqslant 1 /(m(m-2))^{1 / 2}<\pi /(m-1)$ for $m \geqslant 3$. Now the various components of $R$ are separated by angles of $\pi /(m-1)$, so that $f(z) \in R_{m}$. For $m=2$, we have $R_{m}=R$, so that part (a) of the proof is sufficient.

REMARK. It is clear from the inequality $|h(w)| \leqslant 1 / m-h_{1}(1 / m)+\left|h_{1}(w)\right|$ that $|h(w)|=1 / m$ can occur only when $\left|h_{1}(w)\right|=h_{1}(1 / m)$, or $w=1 / m$, if $m>2$, that is, at $z=0$. If $S_{m}=f\left(R_{m}\right)$, then $S_{m} \subset R_{m}$ and the boundaries of $S_{m}$ and $R_{m}$ intersect only at the origin. For $m=2$ this can be verified directly. Indeed the above equality occurs for $w= \pm 1 / m$, which values correspond to $z=0$ and $z=-1$. Both of these points are mapped by $f$ to the origin. One concludes that a sufficiently small translation of $S_{m}$ in the direction of the axis of symmetry of $R_{m}$ will still be a subset of $R_{m}$; that is, if

$$
f_{\varepsilon}=f+\varepsilon \exp \left(\frac{\pi i}{m-1}\right),
$$

then $f_{\varepsilon}\left(\bar{R}_{m}\right) \subset R_{m}$ for all sufficiently small $\varepsilon>0 . f_{\varepsilon}$ has a single fixed point $p_{m}=\varepsilon^{1 / m} \exp (\pi i /(m-1))$ in $R_{m}$.

For a fixed $\varepsilon>0$, let $z_{0}, w_{0} \in R_{m}, z_{n}=f\left(z_{n-1}\right)$, and $w_{n}=f_{\varepsilon}\left(w_{n-1}\right), n=$ $1,2, \ldots$

LEMma 3. For all sufficiently small $\varepsilon>0$, there is an integer $N$ such that, for all $n \geqslant N$,

$$
\left|w_{n+1}-z_{n+1}\right| \leqslant\left|w_{n}-z_{n}\right|\left(1-\frac{1}{2} \varepsilon^{(m-1) / m}\right)+\varepsilon .
$$

Proof. By Lemma $1, w_{n} \rightarrow p_{m}$ as $n \rightarrow \infty$. Choose $N$ such that

$$
w_{n}=p_{m}+t_{n}
$$

with $\left|t_{n}\right|<\varepsilon$ for $n>N$. Also, since $z_{n} \in R_{m}$, we have $z_{n}^{m-1}=-1 / m+r_{m}$, $\left|r_{m}\right|<1 / m$, and

$$
\frac{\pi}{2(m-1)}<\arg z_{n}<\frac{3 \pi}{2(m-1)}
$$


By (4), for $j \geqslant 1$, we have

$$
w_{n}^{j}=p_{m}^{j}+O\left(\varepsilon^{1+(j-1) / m}\right)
$$

as $\varepsilon \rightarrow 0$. Since $\arg p_{m}=\pi /(m-1)$, we have

$$
\arg w_{n}^{j}=\frac{\pi j}{(m-1)}+O\left(\varepsilon^{1-1 / m}\right) .
$$

By (5) and (6), for $1 \leqslant k \leqslant m-1$, we have

$$
\begin{aligned}
\frac{\pi}{2}\left[\frac{m+k-1}{m-1}+O\left(\varepsilon^{1-1 / m}\right)\right] & \leqslant \arg \left(z_{n}^{m-k-1} w_{n}^{k}\right) \\
& \leqslant \frac{3 \pi}{2}\left[\frac{3(m-1)-k}{3(m-1)}+O\left(\varepsilon^{1-1 / m}\right)\right] .
\end{aligned}
$$

It follows that for sufficiently small $\varepsilon>0, \arg \left(z_{n}^{m-k-1} w_{n}^{k}\right)$ and hence also

$$
\arg \left(\sum_{k=1}^{m-2} z_{n}^{m-k-1} w_{n}^{k}\right)=\arg \zeta_{m}
$$

satisfy

$$
\frac{\pi}{2}+\delta \leqslant \arg \zeta_{m} \leqslant \frac{3 \pi}{2}-\delta, \quad \delta>0
$$

Now by (6),

$$
\begin{aligned}
\left|f\left(w_{n}\right)-f\left(z_{n}\right)\right| & =\left|w_{n}-z_{n}\right|\left|1+w_{n}^{m-1}+w_{n}^{m-2} z_{n}+\cdots+z_{n}^{m-1}\right| \\
& \leqslant\left|w_{n}-z_{n}\right|\left\{\left|1-\frac{1}{m}-\varepsilon^{(m-1) / m}+r_{m}+\zeta_{m}\right|+O\left(\varepsilon^{1+(m-2) / m}\right)\right\}
\end{aligned}
$$

and, for sufficiently small $\varepsilon$, by (4) aand (7), we have

$$
\begin{aligned}
\left|\left(1-\frac{1}{m}-\varepsilon^{(m-1) / m}\right)+\zeta_{m}\right|+\left|r_{m}\right| & \leqslant\left|1-\frac{1}{m}-\varepsilon^{(m-1) / m}\right|+\left|r_{m}\right| \\
& \leqslant 1-\varepsilon^{(m-1) / m} .
\end{aligned}
$$

So by (8) and (9),

$$
\begin{aligned}
\left|f\left(w_{n}\right)-f\left(z_{n}\right)\right| & \leqslant\left|w_{n}-z_{n}\right|\left\{\left(1-\varepsilon^{(m-1) / m}\right)+O\left(\varepsilon^{1+(m-2) / m}\right)\right\} \\
& \leqslant\left|w_{n}-z_{n}\right|\left(1-\frac{1}{2} \varepsilon^{(m-1) / m}\right) .
\end{aligned}
$$

The result now follows by the last inequality and the relation

$$
\left|w_{n+1}-z_{n+1}\right| \leqslant\left|f\left(w_{n}\right)-f\left(z_{n}\right)\right|+\varepsilon .
$$

We turn now our attention to the reverse sequence

$$
\zeta_{n}=f^{-1}\left(\zeta_{n-1}\right), \quad n=1,2, \ldots,
$$

where $f^{-1}$ is one of the possible values of the multiple-valued inverse function of $f$. Wherever necessary the exact choice of $f^{-1}$ will be indicated. 
LEMMA 4. The sequence $\left\{\zeta_{n}\right\}_{n=0}^{\infty}$ is bounded for every choice of $\zeta_{0}$. In particular

$$
\left|\zeta_{n}\right| \leqslant \operatorname{Max}\left(2^{1 /(m-1)},\left|\zeta_{0}\right|\right) \text {. }
$$

Proof. Write (10) in the form

$$
\zeta_{n}^{m}+\zeta_{n}=\zeta_{n-1} .
$$

By Cauchy's theorem [2, p. 122], the zeros $\zeta_{n}$ of (12) are bounded in modulus by the only positive zero $r_{n}$ of the polynomial

$$
p(x)=x^{m}-x-r_{n-1}=0, \quad r_{n-1}=\left|\zeta_{n-1}\right| .
$$

(a) If $r_{n-1}>2^{1 /(m-1)}$, then $x^{m}-x>x>r_{n-1}$ for $x>r_{n-1}$. Therefore $r_{n} \leqslant$ $r_{n-1}$.

(b) If $r_{n-1} \leqslant 2^{1 /(m-1)}$, then $r_{n} \leqslant 2^{1 /(m-1)}$ because $p\left(2^{1 /(m-1)}\right) \geqslant 0$. Thus if $\left|\zeta_{0}\right| \leqslant 2^{1 /(m-1)}$, then $\left|\zeta_{n}\right| \leqslant 2^{1 /(m-1)}$, and if $\left|\zeta_{0}\right|>2^{1 /(m-1)}$, then we have (11).

REMARK. Lemma 4 implies that for $K>2^{1 /(m-1)}$, if $\left|\zeta_{0}\right|<K$, then also $\left|\zeta_{n}\right|<K$ for all $n$.

Consider the particular case $m=2$. Let $g(w)=f^{-1}(w)=-\frac{1}{2}+\sqrt{w+\frac{1}{4}}$, where we assume $\operatorname{Im} g(w) \geqslant 0$. If

$$
G_{0}=\{w: \operatorname{Im} w>0\} \cap\{w:|w|<K\}, \quad K>2^{1 /(m-1)},
$$

then $g: G_{0} \rightarrow G_{0}$. The function $g_{\varepsilon}=f+i \varepsilon$ satisfies also $g_{\varepsilon}: G_{0} \rightarrow G_{0}$ for all sufficiently small $\varepsilon>0$ and has the unique fixed point $w_{\varepsilon}=i \varepsilon+\sqrt{i \varepsilon}$ in $G_{0}$. We shall need the following lemma.

Lemma 5. For $\varepsilon>0$ sufficiently small, there is an integer $N$ such that, for all $n \geqslant N$, the sequences $w_{n}=g_{\varepsilon}\left(z_{n-1}\right), z_{0}, w_{0} \in G_{0}, z_{n}=g\left(z_{n-1}\right)$ satisfy

$$
\left|w_{n+1}-z_{n+1}\right| \leqslant M\left|w_{n}-z_{n}\right|+\varepsilon,
$$

where $M=2 /(2+\sqrt{\varepsilon})$.

Proof. Let $\rho_{n}=\left|w_{n}-z_{n}\right|$. Then we have

$$
\rho_{n+1}=\left|\sqrt{w_{n}+\frac{1}{4}}-\sqrt{z_{n}+\frac{1}{4}}+i \varepsilon\right| \leqslant \frac{\rho_{n}}{|A|}+\varepsilon
$$

where $A=\sqrt{w_{n}+\frac{1}{4}}+\sqrt{z_{n}+\frac{1}{4}}$. Let $\sqrt{z_{n}+\frac{1}{4}}=a_{n}+i \alpha_{n}, \sqrt{w_{n}+\frac{1}{4}}=b_{n}+i \beta_{n}$. First we show that $a_{n} \geqslant \frac{1}{2}$ for all $n \geqslant n_{1}$. Indeed, if $z_{n}=x_{n}+i y_{n}$ then we have

$$
\left(2 x_{n+1}+1\right) y_{n+1}=y_{n}
$$

and

$$
x_{n+1}^{2}+x_{n+1}=x_{n}+y_{n+1}^{2} \text {. }
$$


Since $y_{n} \geqslant 0, x_{n+1} \geqslant-\frac{1}{2}$. By (15), $x_{n} \geqslant 0$ implies that $x_{n+1} \geqslant 0$. On the other hand, if $x_{n} \leqslant 0$ for all $n$, then by (14), $y_{n}$ increases to a finite positive limit, say $y_{0}$. (14) then implies that $x_{n} \rightarrow 0$, so that $y_{n} \rightarrow 0$ by (15). Thus $y_{0}=0$, and we have a contradiction.

Secondly, we verify that

$$
\sqrt{w_{\varepsilon}+\frac{1}{4}}=\frac{1}{2}+\sqrt{\frac{1}{2} \varepsilon}+i \sqrt{\frac{1}{2} \varepsilon} .
$$

Since, by Lemma $1, w_{n} \rightarrow w_{\varepsilon}$ as $n \rightarrow \infty$, it follows that, for $n \geqslant n_{2}$, we have

$$
\sqrt{w_{n}+\frac{1}{4}}=\frac{1}{2}+\sqrt{\frac{1}{2} \varepsilon}+i \sqrt{\frac{1}{2} \varepsilon}+O(\varepsilon) .
$$

Thus, for $n \geqslant \operatorname{Max}\left(n_{1}, n_{2}\right)$, we have

$$
\operatorname{Re} A \geqslant 1+\sqrt{\frac{1}{2} \varepsilon}+O(\varepsilon) \geqslant 1+\frac{1}{2} \sqrt{\varepsilon}
$$

for all sufficiently small $\varepsilon$. By (13),

$$
\rho_{n+1} \leqslant M \rho_{n}+\varepsilon,
$$

where $M=2 /(2+\sqrt{\varepsilon})$ and $n \geqslant N(\varepsilon)$. This completes the proof.

REMARK. Solving inequality (16), we obtain for $k=1,2, \ldots$,

$$
\rho_{N+k} \leqslant M^{k} \rho_{N}+\varepsilon \frac{1-M^{k}}{1-M} \leqslant M^{k} \rho_{N}+3 \sqrt{\varepsilon}
$$

\section{The main theorems}

THEOREM 1. For every $z_{0} \in \bar{R}$ the sequence $z_{n+1}=f\left(z_{n}\right)$ converges to zero.

Proof. Assume $z_{0} \in \vec{R}_{m}$. Let $\tau_{n}=\left|w_{n}-z_{n}\right|$. By Lemma 3, for $k=1,2, \ldots$, for $N=N(\varepsilon)$, and for $\varepsilon$ sufficiently small, we have $\tau_{N+k} \leqslant M_{1}^{k} \tau_{N}+$ $\varepsilon\left(1-M_{1}^{k}\right) /\left(1-M_{1}\right)$, where $M_{1}=1-\frac{1}{2} \varepsilon^{(m-1) / m}$. This leads to

$$
\tau_{N+k} \leqslant M_{1}^{k} \tau_{N}+2 \varepsilon^{1 / m} .
$$

By Lemmas 1 and $2,\left\{w_{n}\right\}$ is a convergent sequence, so that $\left|w_{N_{1}+k}-w_{N_{1}+l}\right|<\varepsilon$ for $k, l=1,2, \ldots$, and for $N_{1}$ sufficiently large. Assuming $N_{1} \geqslant N$, we now have, by (18),

$$
\left|z_{N_{1}+k}-z_{N_{1}+l}\right| \leqslant \tau_{N_{1}+k}+\tau_{N_{1}+l}+\varepsilon \leqslant \tau_{N_{1}}\left(M_{1}^{k}+M_{1}^{l}\right)+4 \varepsilon^{1 / m}+\varepsilon .
$$

Therefore

$$
\varlimsup_{m, n \rightarrow \infty}\left|z_{m}-z_{n}\right|=\varlimsup_{k, l \rightarrow \infty}\left|z_{N_{1}+k}-z_{N_{1}+l}\right| \leqslant 4 \varepsilon^{1 / m}+\varepsilon .
$$


Inequality (19) implies that $\left\{z_{n}\right\}$ is a Cauchy sequence and thus converges to the origin as $n \rightarrow \infty$. This completes the proof of Theorem 1 .

THEOREM 2. For every fixed $w_{0} \in C$ the sequence $z_{n+1}=g\left(z_{n}\right)$ tends to zero.

Proof. It is enough to prove Theorem 2 for $w_{0} \in G_{0}$, since the argument carries over for the reflection of $G_{0}$ with respect to the real axis. For real $w_{0}$ the result then follows directly.

By Lemmas 4 and 1, and by (17), we have, for $n \geqslant N_{2}(\varepsilon)$ sufficiently large, and for $k, l=1,2, \ldots$,

$$
\begin{aligned}
\left|z_{N_{2}+k}-z_{N_{2}+l}\right| & \leqslant \rho_{N_{2}+k}+\rho_{N_{2}+l}+\left|w_{N_{2}+k}-w_{N_{2}+l}\right| \\
& \leqslant \rho_{N_{2}}\left(M^{k}+M^{l}\right)+6 \sqrt{\varepsilon}+\varepsilon .
\end{aligned}
$$

Hence

$$
\varlimsup_{m, n \rightarrow \infty}\left|z_{n}-z_{m}\right|=\varlimsup_{l, k \rightarrow \infty}\left|z_{N_{2}+k}-z_{N_{2}+l}\right| \leqslant 6 \sqrt{\varepsilon}+\varepsilon
$$

Therefore $\left\{z_{n}\right\}$ is a convergent sequence and thus tends to the origin.

Corollary. If $m=2$, then for every $z_{0} \in \bar{R}$ there exists a sequence $\left\{z_{n}\right\}_{n=-\infty}^{\infty}$ such that $z_{n+1}=f\left(z_{n}\right)$, and $z_{n} \rightarrow 0, z_{-n} \rightarrow 0$ as $n \rightarrow \infty$. In addition, the sequences $\left\{z_{n}\right\}_{n=0}^{\infty}$ and $\left\{z_{-n}\right\}_{n=0}^{\infty}$ are essentially disjoint (except for a finite number of elements).

Proof. This is a direct result of Theorems 1 and 2, and of the relations $\operatorname{Re} z_{-n} \geqslant 0$ for $n \geqslant n_{1}$, and $\operatorname{Re} z_{n}<0$ for $n \geqslant 0$, if $z_{0} \neq 0$.

We conclude with two conjectures.

CONJECTURE 1. Let $f(z)=z+z^{m}, m \geqslant 2$. There exists a determination of $f^{-1}(z)$ such that for every $z_{0} \in C$ the sequence $z_{n}=f^{-1}\left(z_{n-1}\right)$ tends to zero as $n \rightarrow \infty$.

If this conjecture is true, then by the previous results it would be possible to construct cyclic sequences for a polynomial of arbitrary degree $m \geqslant 2$.

CONJECTURE 2. Let $f(z)=z+a_{2} z^{2}+\cdots+a_{m} z^{m}$ be of degree $m \geqslant 2$, and assume that $a_{k} \geqslant 0$ for all $k$. Then for every $z_{0}$ such that $\left|f^{\prime}\left(z_{0}\right)\right| \leqslant 1$, the sequence $z_{n+1}=f\left(z_{n}\right)$ converges. 


\section{Acknowledgement}

The author thanks the referee for several valuable suggestions.

\section{References}

[1] I. N. Baker and Z. Rubinstein, 'Simultaneous iteration by entire or rational functions and their inverses', J. A ustral. Math. Soc. Ser. A 34 (1983), 364-367.

[2] M. Marden, 'Geometry of polynomials', (Mathematical Surveys Number 3, Amer. Math. Soc., Providence, R.I., 1966).

[3] Peter L. Walter, 'Iterated complex radicals', The Mathematical Gazette 67 (1983), 269-273.

Department of Mathematics

University of Colorado

Boulder, Colorado 80309

U.S.A.
Department of Mathematics

University of Haifa

Mount Carmel, Haifa

Israel 